\title{
The Effect of Vascular Risk Factors on the Efficacy of Rivastigmine Patch and Capsule Treatment in Alzheimer's Disease
}

\author{
M.R. Farlow ${ }^{a}$ P.M. Doraiswamy ${ }^{b} \quad$ X. Meng ${ }^{c} \quad$ K. Cooke $^{d} \quad$ M. Somogyi ${ }^{c}$ \\ ${ }^{a}$ Department of Neurology, Indiana University School of Medicine, Indianapolis, Ind., \\ ${ }^{b}$ Department of Psychiatry and Medicine, Duke University Medical Center, Durham, N.C., \\ ${ }^{\mathrm{C} N o v a r t i s}$ Pharmaceuticals Corporation, East Hanover, N.J., USA; ${ }^{\mathrm{d}}$ Alpha-Plus Medical \\ Communications Ltd., Abingdon, Oxfordshire, UK
}

\section{Key Words}

Alzheimer's disease $\cdot$ Efficacy $\cdot$ Rivastigmine $\cdot$ Transdermal patch $\cdot$ Vascular risk factors

\begin{abstract}
Background: Vascular risk factors (VRF) may influence response to rivastigmine in Alzheimer's disease (AD). Methods: AD patients who participated in a randomized, double-blind, placebocontrolled trial of rivastigmine patch and capsule treatment were stratified by baseline VRF status. Treatment response was evaluated using the AD Assessment Scale-cognitive subscale (ADAS-cog), AD Cooperative Study-Clinical Global Impression of Change (ADCS-CGIC) and the AD Cooperative Study-Activities of Daily Living (ADCS-ADL) scale. Results: ADAS-cog scores significantly improved in all rivastigmine-treated patients ( $p<0.05$ vs. placebo), except 9.5 $\mathrm{mg} / 24 \mathrm{~h}$ patch-treated patients with VRF, and were significantly affected by VRF status in the study population as a whole. Significant benefits were seen on the ADCS-ADL in $9.5 \mathrm{mg} / 24 \mathrm{~h}$ patch- and capsule-treated patients with, but not without, VRF. The ADCS-CGIC significantly improved in capsule-treated patients with, and patch-treated patients without VRF. Although non-significant, patients without VRF showed an apparent faster rate of placebo decline. Conclusion: VRF may influence AD progression and response to rivastigmine.
\end{abstract}




\section{Introduction}

The cholinesterase inhibitor rivastigmine is approved in the USA and a number of other countries worldwide for the symptomatic treatment of mild-to-moderate Alzheimer's disease (AD). Rivastigmine is available as oral capsules, but it is also the first AD therapy to be licensed as a transdermal patch.

The rivastigmine transdermal patch provides steady plasma concentrations of rivastigmine and bypasses first-pass metabolism in the gut and liver [1]. The efficacy of oral rivastigmine is thought to be dose-dependent $[2,3]$. However, oral rivastigmine treatment may be associated with a number of gastrointestinal side effects, such as nausea and vomiting, which may limit the maximum therapeutic dose that can be achieved by some patients [4].

A large, international, 24-week, multicenter, randomized, double-blind, placebo- and active-controlled trial (Investigation of transDermal Exelon in ALzheimer's disease; IDEAL) compared the efficacy, safety, and tolerability of rivastigmine patch $\left(9.5 \mathrm{mg} / 24 \mathrm{~h}\left[10 \mathrm{~cm}^{2}\right]\right.$ and $17.4 \mathrm{mg} / 24 \mathrm{~h}\left[20 \mathrm{~cm}^{2}\right]$ ), rivastigmine capsules (12 $\mathrm{mg} /$ day), and placebo [5]. The rivastigmine $9.5 \mathrm{mg} / 24 \mathrm{~h}$ patch was associated with comparable efficacy to $12 \mathrm{mg} /$ day capsules, but superior tolerability, as there were approximately two-thirds fewer reports of gastrointestinal adverse events in the patch group than the capsule group [5]. This improved tolerability may allow access to higher doses, leading to enhanced treatment benefits $[6,7]$.

The concept of 'responders' and 'non-responders' to cholinesterase inhibitor therapy has been discussed for a number of years, and has a variable impact on the efficacy of treatment reported in clinical trials [8]. Suboptimal dosing may be associated with a lower probability of showing a response to cholinesterase inhibitor therapy, reflecting the dose-response relationship seen with these agents $[2,3]$. As well as dose, it has been suggested that a number of other variables, including age [9], disease stage [10,11], and the presence of vascular risk factors (VRF) [12, 13], may influence the response to cholinesterase inhibitors such as rivastigmine. To date, associations between the presence of VRF and prediction of response to rivastigmine have been based on trials with oral capsules $[12,13]$. The IDEAL study provides us with a large database whereby similar analyses may be performed to investigate whether the presence of concurrent VRF may predict response to transdermal as well as oral rivastigmine.

\section{Methods}

This was a retrospective analysis of a large, international, 24-week, randomized, doubleblind, placebo- and active-controlled trial (CENA713D2320, IDEAL) which investigated the efficacy, safety, and tolerability of rivastigmine capsule $(12 \mathrm{mg} /$ day), rivastigmine patch $(9.5$ and $17.4 \mathrm{mg} / 24 \mathrm{~h}$ ), and placebo [5].

The full details of the IDEAL study have been published previously [5]. Briefly, patients were 50-85 years of age, with a diagnosis of probable AD (DSM-IV, NINCDS/ADRDA criteria) $[14,15]$ and a Mini-Mental State Examination (MMSE) score of 10-20 [16]. Patients were excluded from study entry if they had: any form of advanced, progressive or unstable disease that may have affected safety, or the ability to complete assessments; a diagnosis of any condition other than $\mathrm{AD}$ that may cause dementia (e.g., vascular dementia); used any new psychotropic or dopaminergic agents, cholinesterase inhibitors, or centrally acting cholinergic agents during the 4 weeks prior to randomization; a history (past year) or existing diagnosis of cerebrovascular disease, e.g., stroke, transient ischemic attack, and/or aneurysms, and an existing diagnosis of severe cardiovascular disease. 
A brain scan (magnetic resonance imaging) was performed at screening, if a scan had not been performed in the previous 12 months, to ensure patients met the entry criteria and exclude patients with probable vascular dementia. Patients with a score $>4$ on the Modified Hachinski Ischemia Scale (suggestive of vascular dementia) were excluded. Laboratory tests (total cholesterol and homocysteine) were performed at screening, providing information on VRF in the study participants.

Study participants were randomized to receive $12 \mathrm{mg} /$ day rivastigmine capsules, $9.5 \mathrm{mg} / 24 \mathrm{~h}$ rivastigmine patch, $17.4 \mathrm{mg} / 24 \mathrm{~h}$ rivastigmine patch, or placebo. Patients were up-titrated to their target dose in 4 -week steps over a 16-week period and then maintained at their target dose for a further 8 weeks. Protocols and study materials were reviewed by the responsible overseeing institutional review board for each site/country. All subjects provided informed consent before participating in the study. The study was conducted according to the ethical principles of the Declaration of Helsinki, as revised in 2000.

Primary outcome measures included the change from baseline at week 24 on the AD Assessment Scale-cognitive subscale (ADAS-cog) [17] and the week-24 AD Cooperative Study-Clinical Global Impression of Change (ADCS-CGIC) score [18]. Secondary outcome measures included the change from baseline at week 24 on the ADCS-Activities of Daily Living (ADCS-ADL) scale [19]. Safety evaluations included monitoring vital signs and skin tolerability, and reporting of all adverse events. Efficacy analyses were based on the intentto-treat (ITT) principle, with all patients who had received at least 1 dose of study medication and provided at least 1 baseline and 1 post-baseline efficacy assessment included in the analyses [ITT last observation carried forward (LOCF) population].

In the current analysis, patients in the ITT-LOCF population were stratified, relative to their allocated treatment group, according to whether their case report forms documented $\geq 1$ active VRF at screening. VRF were active at study entry. VRF reported in the study population at screening included: angina pectoris; arteriosclerosis; atherosclerosis; atrial fibrillation; raised blood homocysteine; carotid artery disease; carotid artery stenosis; cerebral artery embolism; cerebral atherosclerosis; cerebral ischemia; decreased cerebral perfusion pressure; coronary angioplasty; coronary artery atherosclerosis; coronary artery disease; diabetes mellitus; insulin-dependent diabetes mellitus; non-insulin-dependent diabetes mellitus; diabetic microangiopathy; essential hypertension; hypercholesterolemia; hyperglycemia; hyperlipidemia; hypertension; hypertensive heart disease; hypertriglyceridemia; myocardial infarction; myocardial ischemia; obesity; peripheral ischemia; peripheral occlusive disease; peripheral vascular disorder; quadruple-vessel bypass graft, and vascular and venous insufficiency.

The mean change [least-square (LS) means] from baseline at week 24 on the ADAS-cog (total, domains and individual items) and ADCS-ADL, and week-24 ADCS-CGIC scores (LS means) were assessed relative to treatment and VRF status for the ITT-LOCF population. Effect sizes were calculated using Cohen's d for each of the individual ADAS-cog items. The statistical significance of treatment differences on the ADAS-cog and ADCS-ADL was assessed using an analysis of covariance (ANCOVA) model, with treatment, VRF, treatment by VRF, and country as factors, and baseline scores as a covariate. Treatment differences on the ADCS-CGIC were assessed using an ANCOVA model, with treatment, VRF, treatment by VRF and country as factors. Additional evaluations included the effect of VRF on the incidence of adverse events and the rate of placebo decline. Analysis of placebo decline was based on the ITT observed case (ITT-OC) population and compared using an analysis of variance (ANOVA) model. 
Farlow et al.: Rivastigmine in Alzheimer Patients with Vascular Risk Factors

\section{Results}

\section{Patients}

In total, 1195 patients were entered in the IDEAL study: 293 were randomized to receive the rivastigmine $9.5 \mathrm{mg} / 24 \mathrm{~h}$ patch; 303 to the rivastigmine $17.4 \mathrm{mg} / 24 \mathrm{~h}$ patch; 297 to the rivastigmine $12 \mathrm{mg} /$ day capsule and 302 to placebo. Of these patients, 636 were defined as having concurrent VRF at baseline, based on their medical history and 'active' comorbid conditions reported during the screening visit.

The baseline characteristics of the study population are summarized in table 1. Patients in the rivastigmine-treated and placebo groups were comparable at baseline in terms of their gender, race and scores on the MMSE, ADAS-cog and ADCS-ADL scales. However, on average, patients with concurrent VRF tended to be slightly older and have a higher body weight than patients without concurrent VRF at baseline (table 1).

The loading dose of the $9.5 \mathrm{mg} / 24 \mathrm{~h}$ patch is $18 \mathrm{mg}$ and the $17.4 \mathrm{mg} / 24 \mathrm{~h}$ patch has a loading dose of $36 \mathrm{mg}$ [20]. The last prescribed rivastigmine dose was calculated, in terms of loading dose, for patients with and without concurrent VRF (safety population). The mean (standard deviation; SD) last prescribed doses for patients with concurrent VRF in the $9.5 \mathrm{mg} / 24 \mathrm{~h}$ patch, $17.4 \mathrm{mg} / 24 \mathrm{~h}$ patch, and $12 \mathrm{mg}$ /day capsule groups were 17.0 (2.8), 28.8 (10.2), and 9.3 (3.6) $\mathrm{mg}$, respectively. For patients without concurrent VRF at baseline, the mean (SD) last prescribed doses for the $9.5 \mathrm{mg} / 24 \mathrm{~h}$ patch, $17.4 \mathrm{mg} / 24 \mathrm{~h}$ patch and the $12 \mathrm{mg} /$ day capsule groups were 17.3 (2.4), 27.7 (9.4), and 9.2 (3.5) mg, respectively.

\section{Rate of Placebo Decline}

There was an apparent faster decline in the placebo group from baseline at week 24 on the ADAS-cog in patients who did not show signs of concurrent VRF at baseline compared with patients with concurrent VRF (fig. 1). However, the observed differences did not reach significance $(\mathrm{p}=0.154)$.

\section{Efficacy}

Rivastigmine-treated patients showed significant improvements from baseline at week 24 on the ADAS-cog (table 2). When stratifying patients by their VRF status, significant improvements were seen from baseline at week 24 on the ADAS-cog in rivastigmine $17.4 \mathrm{mg} / 24 \mathrm{~h}$ patch- and capsule-treated patients with concurrent VRF, and in all rivastigmine-treated groups for patients without concurrent VRF (all $\mathrm{p}<0.05$ vs. placebo; table 2; fig. 1). ADAS-cog performance did not differ significantly between patients with and without VRF in the individual treatment groups (all $\mathrm{p}>0.05$ vs. placebo; table 2). However, significant differences in ADAS-cog performance were seen between patients with and without concurrent VRF when comparing the study population as a whole $(\mathrm{p}=0.041)$.

Across the whole study population, the mean change from baseline at week 24 on the ADAS-cog language domain was significantly different between patients with and without concurrent VRF ( $\mathrm{p}=0.005)$. When considering the individual treatment groups, significant differences on the ADAS-cog language domain were observed between patients with and without concurrent VRF randomized to receive placebo $(\mathrm{p}=0.010)$, with patients without concurrent VRF showing an apparent faster rate of placebo decline. Of the individual items that comprise the language domain, significant differences were observed between patients with and without concurrent VRF on items that assess commands, spoken language ability and word finding difficulty $(\mathrm{p}=0.024, \mathrm{p}=0.0008$, and $\mathrm{p}=0.030$, respectively). These differences also reached significance on the spoken language ability item for capsule-treated patients ( $\mathrm{p}=0.033$ VRF vs. no VRF) and the spoken language ability, word finding difficulty, and comprehension items for patients who received placebo $(p=0.044, p=0.028, p=0.020$ 
Fig. 1. Mean change from baseline at week 24 on the ADAS-cog by treatment and VRF status (ITT-LOCF population). * $\mathrm{p}<$ 0.05 vs. placebo, ${ }^{* *} \mathrm{p}<0.01$ vs. placebo (LS means).

Farlow et al.: Rivastigmine in Alzheimer Patients with Vascular Risk Factors

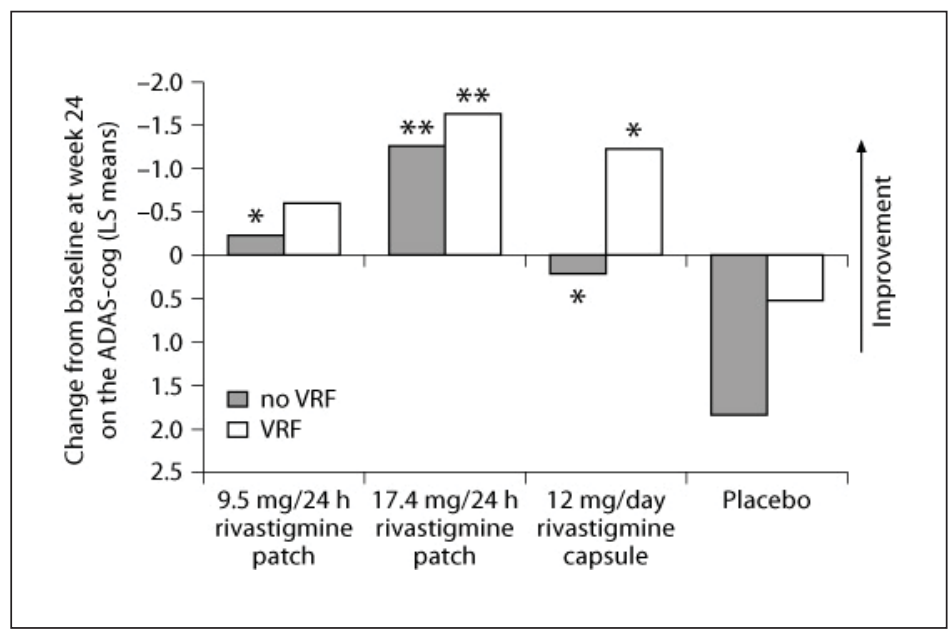

Fig. 2. Effect sizes for each of the individual ADAS-cog items by treatment and VRF status.

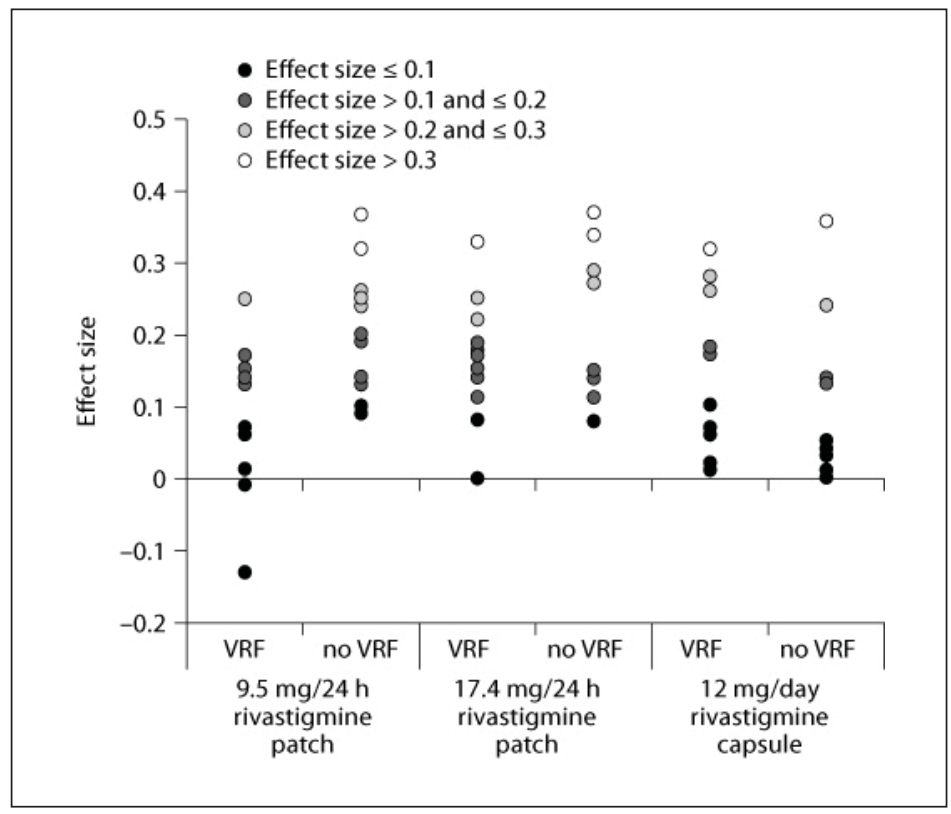

VRF vs. no VRF, respectively). VRF status was not shown to have any significant effect on the ADAS-cog memory ( $\mathrm{p}=0.211 \mathrm{VRF}$ vs. no VRF) or praxis $(\mathrm{p}=0.362 \mathrm{VRF}$ vs. no VRF) domains, or the items which comprise these domains (with the exception of word recall amongst patients who received placebo, $\mathrm{p}=0.035 \mathrm{VRF}$ vs. no VRF).

Effect sizes were calculated for each of the individual ADAS-cog items by treatment group and VRF status. When comparing the magnitude of the effect sizes between patients with and without concurrent VRF, there was an overall trend toward a greater effect size on the individual ADAS-cog items in patients without concurrent VRF (fig. 2). Patch-treated patients without concurrent VRF had fewer items with an effect size $<0.1$, and more items with an effect size $>0.2$ than patch-treated patients with concurrent VRF (fig. 2).

Rivastigmine patch- and capsule-treated patients also showed significant improvements at week 24 on the ADCS-CGIC ( $p<0.05$ vs. placebo; table 2). When taking the patients' VRF 
Farlow et al.: Rivastigmine in Alzheimer Patients with Vascular Risk Factors

Table 2. Mean change from baseline at week 24 on the ADAS-cog and ADCS-ADL, and mean week-24 ADCS-CGIC score by treatment and VRF status (ITT-LOCF population)

\begin{tabular}{|c|c|c|c|c|}
\hline & \multicolumn{3}{|l|}{ Rivastigmine } & \multirow[t]{2}{*}{ Placebo } \\
\hline & $\begin{array}{l}9.5 \mathrm{mg} / 24 \mathrm{~h} \\
\text { patch }\end{array}$ & $\begin{array}{l}17.4 \mathrm{mg} / 24 \mathrm{~h} \\
\text { patch }\end{array}$ & $\begin{array}{l}12 \mathrm{mg} / \text { day } \\
\text { capsule }\end{array}$ & \\
\hline \multicolumn{5}{|l|}{ All patients } \\
\hline ADAS-cog, n & 248 & 262 & 253 & 281 \\
\hline Mean \pm SD & $-0.59 \pm 6.37$ & $-1.60 \pm 6.53$ & $-0.62 \pm 6.22$ & $0.97 \pm 6.80$ \\
\hline p value vs. placebo ${ }^{a}$ & 0.005 & $<0.0001$ & 0.003 & - \\
\hline ADCS-CGIC, $\mathrm{n}$ & 248 & 260 & 253 & 278 \\
\hline Mean \pm SD & $3.91 \pm 1.20$ & $3.96 \pm 1.27$ & $3.91 \pm 1.25$ & $4.19 \pm 1.26$ \\
\hline p value vs. placebo ${ }^{a}$ & 0.012 & 0.022 & 0.010 & - \\
\hline ADCS-ADL, n & 247 & 263 & 254 & 281 \\
\hline Mean \pm SD & $-0.14 \pm 9.07$ & $-0.02 \pm 11.60$ & $-0.48 \pm 9.50$ & $-2.27 \pm 9.36$ \\
\hline p value vs. placebo $^{a}$ & 0.017 & 0.016 & 0.040 & - \\
\hline \multicolumn{5}{|c|}{ Patients with concurrent VRF (VRF) } \\
\hline ADAS-cog, $n$ & 141 & 138 & 129 & 155 \\
\hline Mean \pm SD & $-0.66 \pm 6.44$ & $-1.72 \pm 6.58$ & $-1.32 \pm 5.90$ & $0.45 \pm 6.82$ \\
\hline p value vs. placebo ${ }^{a}$ & 0.130 & 0.004 & 0.023 & - \\
\hline p value VRF vs. no VRF & 0.663 & 0.644 & 0.083 & 0.096 \\
\hline ADCS-CGIC, n & 140 & 136 & 128 & 153 \\
\hline Mean \pm SD & $3.90 \pm 1.17$ & $3.99 \pm 1.26$ & $3.78 \pm 1.24$ & $4.12 \pm 1.35$ \\
\hline p value vs. placebo ${ }^{a}$ & 0.147 & 0.411 & 0.029 & - \\
\hline p value VRF vs. no VRFa & 0.672 & 0.735 & 0.071 & 0.195 \\
\hline ADCS-ADL, $\mathrm{n}$ & 140 & 138 & 130 & 155 \\
\hline Mean \pm SD & $0.16 \pm 8.69$ & $-0.19 \pm 12.36$ & $0.09 \pm 10.11$ & $-2.39 \pm 10.15$ \\
\hline p value vs. placebo ${ }^{a}$ & 0.027 & 0.116 & 0.041 & - \\
\hline p value VRF vs. no VRF ${ }^{a}$ & 0.313 & 0.925 & 0.200 & 0.783 \\
\hline \multicolumn{5}{|c|}{ Patients without concurrent VRF (no VRF) } \\
\hline ADAS-cog, $\mathrm{n}$ & 107 & 124 & 124 & 126 \\
\hline Mean \pm SD & $-0.49 \pm 6.31$ & $-1.48 \pm 6.50$ & $0.10 \pm 6.47$ & $1.61 \pm 6.75$ \\
\hline p value vs. placebo ${ }^{a}$ & 0.014 & 0.0001 & 0.046 & - \\
\hline ADCS-CGIC, $\mathrm{n}$ & 108 & 124 & 125 & 125 \\
\hline Mean \pm SD & $3.93 \pm 1.25$ & $3.92 \pm 1.28$ & $4.05 \pm 1.26$ & $4.28 \pm 1.15$ \\
\hline p value vs. placebo ${ }^{a}$ & 0.038 & 0.019 & 0.135 & - \\
\hline ADCS-ADL, n & 107 & 125 & 124 & 126 \\
\hline Mean \pm SD & $-0.53 \pm 9.57$ & $0.17 \pm 10.75$ & $-1.08 \pm 8.82$ & $-2.11 \pm 8.32$ \\
\hline p value vs. placebo $^{a}$ & 0.224 & 0.069 & 0.375 & - \\
\hline
\end{tabular}

status into consideration, rivastigmine capsule-treated patients with concurrent VRF and patch-treated ( 9.5 and $17.4 \mathrm{mg} / 24 \mathrm{~h}$ ) patients without concurrent VRF showed significant improvements ( $\mathrm{p}=0.029, \mathrm{p}=0.038, \mathrm{p}=0.019$ vs. placebo, respectively) at week $24 \mathrm{on}$ the ADCS-CGIC (table 2; fig. 3). Despite rivastigmine $9.5 \mathrm{mg} / 24 \mathrm{~h}$ patch- and $12 \mathrm{mg} /$ day capsule-treated patients with concurrent VRF showing a trend towards better performance on the ADCS-CGIC than patients without concurrent VRF, VRF status did not have a significant effect on week 24 ADCS-CGIC scores $(\mathrm{p}=0.672, \mathrm{p}=0.071$ VRF vs. no VRF; respectively; table 2; fig. 3 ). 
Fig. 3. Mean ADCS-CGIC score at week 24 by treatment and VRF status (ITT-LOCF population). ${ }^{*} \mathrm{p}<0.05$ vs. placebo (LS means).

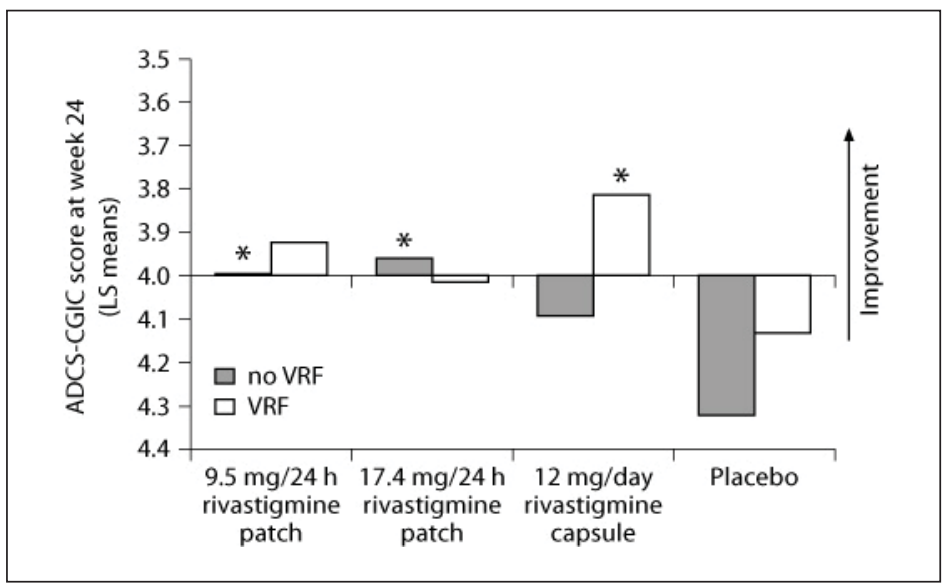

Fig. 4. Mean change from baseline at week 24 on the ADCSADL by treatment and VRF status (ITT-LOCF population). ${ }^{*} \mathrm{p}<0.05$ vs. placebo (LS means).

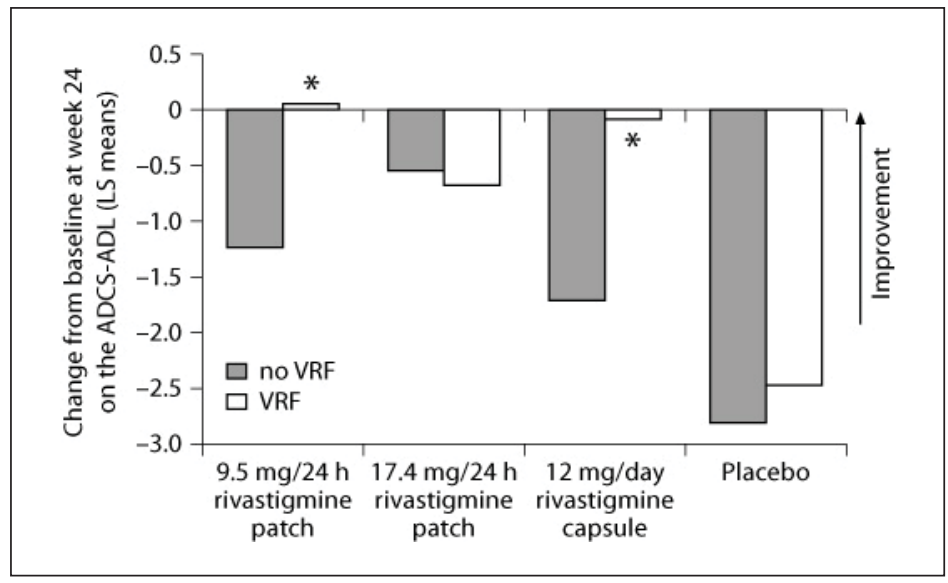

Significant benefits of treatment were seen on the ADCS-ADL from baseline at week 24 in rivastigmine patch- and capsule-treated patients compared with placebo ( $\mathrm{p}<0.05$; table 2). More specifically, rivastigmine $9.5 \mathrm{mg} / 24 \mathrm{~h}$ patch- and capsule-treated patients with concurrent VRF, but not those without, showed significant changes from baseline at week 24 on the ADCS-ADL ( $\mathrm{p}=0.027, \mathrm{p}=0.041$ vs. placebo, respectively; table 2 ; fig. 4). Rivastigmine $9.5 \mathrm{mg} / 24 \mathrm{~h}$ patch- and capsule-treated patients with concurrent VRF showed a numerical trend towards better performance on the ADCS-ADL than patients without concurrent VRF (fig. 4). However, these differences did not reach significance ( $p>0.05$ VRF vs. no VRF) across any of the treatment groups (table 2), or in the study population as a whole.

\section{Safety}

Overall, there was a significant difference in the incidence of adverse events between patients with and without concurrent VRF (61.5 and 51.2\%, respectively; $\mathrm{p}=0.0004 \mathrm{VRF}$ vs. no VRF; table 3). In each of the individual treatment groups, the overall incidence of adverse events was slightly higher in those patients who had concurrent VRF at baseline compared with those without ( 58.9 vs. $40.6 \%$ in the $9.5 \mathrm{mg} / 24 \mathrm{~h}$ patch group, $68.4 \mathrm{vs} .63 .5 \%$ in the 17.4 $\mathrm{mg} / 24 \mathrm{~h}$ patch group, and $68.0 \mathrm{vs.} 58.5 \%$ in the $12 \mathrm{mg}$ /day capsule group, respectively; table 3 ). 


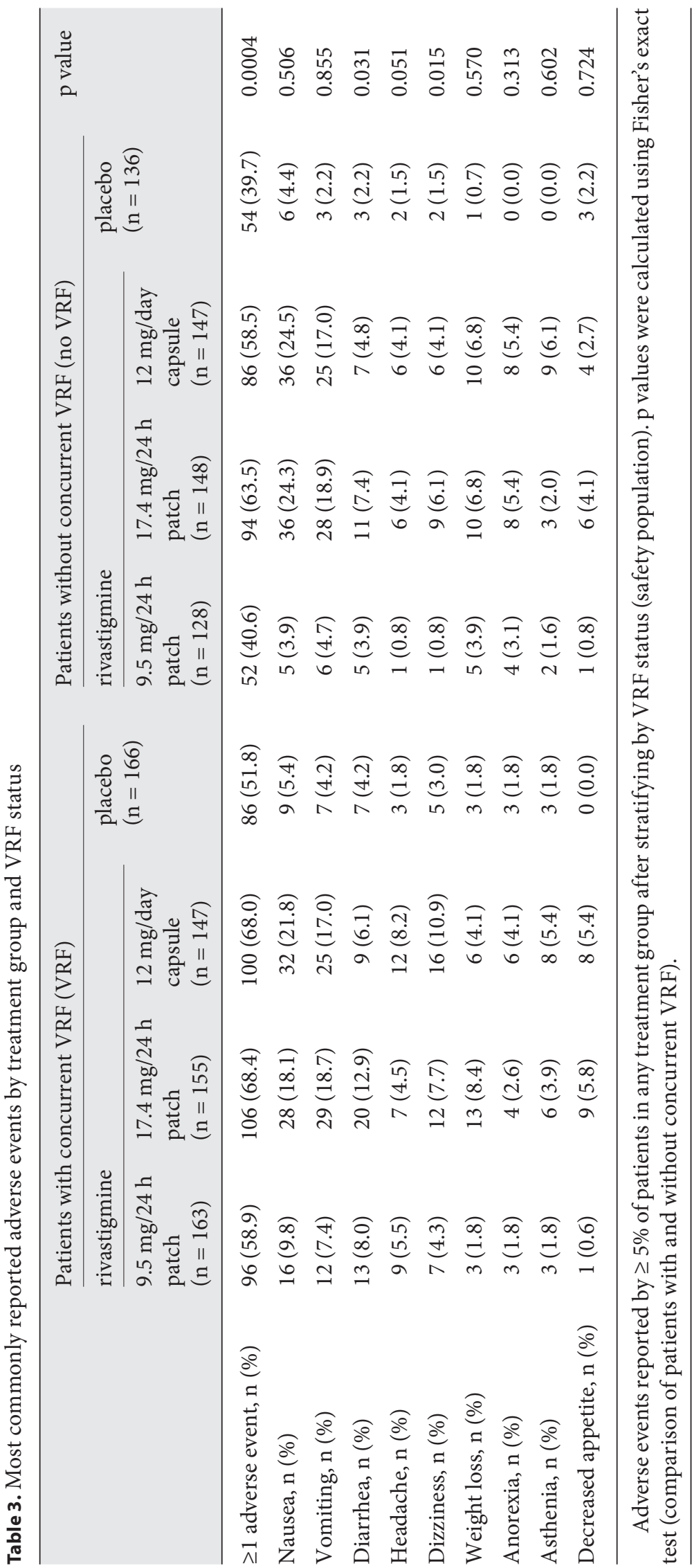


However, the same trend was also seen in patients in the placebo group (51.8 vs. $39.7 \%$, respectively; table 3). Irrespective of VRF status, the most common adverse events reported by rivastigmine-treated patients were cholinergic in nature, including nausea, vomiting, and diarrhea (table 3). The incidence of nausea and vomiting tended to be lower in patients with and without concurrent VRF treated with the $9.5 \mathrm{mg} / 24 \mathrm{~h}$ patch compared with $12 \mathrm{mg} / \mathrm{day}$ capsules (table 3 ). Of the adverse events reported by $>5 \%$ of patients in any treatment group after stratifying by VRF status, the incidence of diarrhea and dizziness were significantly different when comparing patients with and without concurrent VRF ( $\mathrm{p}<0.05$ VRF vs. no VRF; table 3).

\section{Discussion}

In this retrospective analysis of the IDEAL study, we investigated the effect of VRF on the response to oral and transdermal rivastigmine. Significant improvements were seen on the ADAS-cog (compared with placebo) in all rivastigmine-treated patients, except for 9.5 $\mathrm{mg} / 24 \mathrm{~h}$ patch-treated patients with concurrent VRF. Significant treatment benefits were observed on the ADCS-ADL in $9.5 \mathrm{mg} / 24 \mathrm{~h}$ patch- and capsule-treated patients with, but not without, concurrent VRF. ADCS-CGIC scores significantly improved in capsule-treated patients with and patch-treated patients without VRF. VRF status had a significant effect on ADAS-cog performance when comparing the study population as a whole, but no significant effects on efficacy were observed when comparing the individual treatment groups. However, VRF status was shown to have a significant effect on performance on the language domain of the ADAS-cog, and 3 of 4 items (commands, word finding difficulty, and spoken language ability) that comprise this domain. Although these findings require further investigation, they suggest that VRF may impact on language aspects of cognition, in terms of the rate of decline and the degree of symptomatic improvement observed over 24 weeks.

Overall, although non-significant, VRF status appeared to have a greater influence on the efficacy (total ADAS-cog, ADCS-CGIC, and ADCS-ADL scores) of oral than transdermal rivastigmine. These observations suggest that the higher peak plasma concentrations of rivastigmine observed with oral compared with transdermal treatment may offer some benefits in patients with VRF, despite the greater potential for adverse events.

Other studies have reported that rivastigmine capsule treatment is associated with greater efficacy in patients with concurrent VRF $[12,13]$. A 104-week, open-label extension of a 26-week, placebo-controlled trial of rivastigmine capsule treatment in patients with mild-to-moderate $\mathrm{AD}$ found that hypertensive $\mathrm{AD}$ patients who had received rivastigmine capsules during both phases of the study showed greater benefits of treatment than patients who had switched to rivastigmine (from placebo) at the start of the open-label extension. In contrast, treatment duration did not appear to have any significant effect on the efficacy of rivastigmine treatment in non-hypertensive patients [12]. A study examining the efficacy, safety, and tolerability of rivastigmine capsules in patients with and without concurrent VRF found that rivastigmine was equally safe and well-tolerated in both groups of patients, but patients with concurrent VRF showed increased benefits of treatment on cognition and ADL [13]. A further study has demonstrated that treatment with oral rivastigmine is associated with efficacy in patients both with and without concurrent hypertension [21]. However, treatment with 6-12 mg/day rivastigmine capsules was associated with significantly greater efficacy on the ADAS-cog than 1-4 mg/day rivastigmine capsules in hypertensive but not non-hypertensive patients [21].

Although non-significant, patients without concurrent VRF appeared to show a trend towards a faster rate of placebo decline on the ADAS-cog than patients with concurrent VRF. 
A similar trend ( $p$ values not reported) was observed in the ADAS-cog data from a study examining the effect of concurrent arterial hypertension on the efficacy of rivastigmine capsules in $\mathrm{AD}$ patients [21]. In the current analysis, we observed a shift towards a greater effect size on individual ADAS-cog items in rivastigmine-treated patients without concurrent VRF. The trend towards greater placebo decline observed in patients without concurrent VRF on the total ADAS-cog and some items may have increased the effect size in these patients, as effect sizes take into account drug-placebo differences. These findings may be interesting to investigate further, as they suggest that there may be differences in the brain physiology of patients with VRF that may influence disease progression.

Imaging studies have shown that patients who respond to rivastigmine show increased frontal, temporal, and parietal regional cerebral blood flow when compared with untreated patients or 'non-responders' $[22,23]$. These increases in blood flow have been correlated with improvements in cognition, memory, and attention. These findings may explain, in part, why patients with VRF, who may have high blood pressure, damaged blood vessels, and physiological changes in blood flow, appear to demonstrate an enhanced response to rivastigmine.

This analysis was designed to investigate the efficacy, safety, and tolerability of oral and transdermal rivastigmine in patients with mild-to-moderate $\mathrm{AD}$ and concurrent VRF. This was a retrospective analysis of one grouping of VRF that was limited by multiple comparisons and lack of direct imaging data on brain ischemic changes, and hence was only intended to be hypothesis forming. No corrections were made for multiple comparisons due to the exploratory nature of the analyses. The IDEAL study was not powered to detect treatment differences between AD patients with and without concurrent VRF at baseline. These factors impact on the robustness of the data, and must be taken into consideration when interpreting the results.

In conclusion, these data suggest that rivastigmine treatment may benefit patients both with and without concurrent VRF. However, VRF status may impact on aspects of the observed treatment response, such as cognition, and more specifically, language. Larger studies are needed to confirm these findings and fully investigate the effect of concurrent VRF on the efficacy, safety, and tolerability of rivastigmine in mild-to-moderate AD. In particular, the effect of VRF on the rate of placebo decline and disease progression warrants further investigation, as this may be clinically important when considering optimal treatment strategies for patients with a number of comorbid conditions.

\section{Acknowledgments}

The development of this paper was supported by Novartis Pharmaceuticals Corporation. Novartis developed and manufactured rivastigmine, and sponsored the large, multicenter, randomized, double-blind trials that led to the approval of rivastigmine in the USA for mild-to-moderate $\mathrm{AD}$.

\section{Disclosure Statement}

Martin R. Farlow has provided expert consultation services and received speaker honoraria and research funding from Novartis Pharmaceuticals Corporation. P. Murali Doraiswamy has received research grants and served as a paid advisor to several companies in the field, including Novartis. He also owns stocks in Sonexa Therapeutics. Xiangyi Meng and Monique Somogyi are full-time employees and stock holders of Novartis Pharmaceuti- 
cals Corporation. The development of this manuscript was supported by Novartis Pharmaceuticals Corporation. Medical writing and editorial assistance was provided by Katy Cooke of Alpha-Plus Medical Communications Limited (UK), and this service was supported by Novartis Pharmaceuticals Communications.

\section{References}

-1 Oertel W, Ross J, Eggert K, Adler G: Rationale for transdermal drug administration in Alzheimer's disease. Neurology 2007;69(suppl 1):S4-S9.

-2 Schneider LS, Anand R, Farlow MR: Systematic review of the efficacy of rivastigmine for patients with Alzheimer's disease. Int J Geriatr Psychopharmacol 1998;1:S26-S34.

-3 Anand R, Messina J, Hartman R: Dose-response effect of rivastigmine in the treatment of Alzheimer's disease. Int J Geriatr Psychopharmacol 2000;2:68-72.

4 Corey-Bloom J, Anand R, Veach J: A randomized trial evaluating the efficacy and safety of ENA 713 (rivastigmine tartrate), a new acetylcholinesterase inhibitor, in patients with mild to moderately severe Alzheimer's disease. Int J Geriatr Psychopharmacol 1998;1:55-65.

- 5 Winblad B, Cummings J, Andreasen N, Grossberg G, Onofrj M, Sadowsky C, Zechner S, Nagel J, Lane R: A six-month double-blind, randomized, placebo-controlled study of a transdermal patch in Alzheimer's disease - rivastigmine patch versus capsule. Int J Geriatr Psychiatry 2007;22:456-467.

-6 Kurz A, Farlow M, Lefevre G: Pharmacokinetics of a novel transdermal rivastigmine patch for the treatment of Alzheimer's disease: a review. Int J Clin Pract 2009;63:799-805.

7 Small G, Dubois B: A review of compliance to treatment in Alzheimer's disease: potential benefits of a transdermal patch. Curr Med Res Opin 2007;23:2705-2713.

-8 Raschetti R, Maggini M, Sorrentino GC, Martini N, Caffari B, Vanacore N: A cohort study of effectiveness of acetylcholinesterase inhibitors in Alzheimer's disease. Eur J Clin Pharmacol 2005;61: 361-368.

-9 Bullock R, Bergman H, Touchon J, Gambina G, He Y, Nagel J, Lane R: Effect of age on response to rivastigmine or donepezil in patients with Alzheimer's disease. Curr Med Res Opin 2006;22:483494.

-10 Burns A, Spiegel R, Quarg P: Efficacy of rivastigmine in subjects with moderately severe Alzheimer's disease. Int J Geriatr Psychiatry 2004;19:243-249.

-11 Kurz A, Farlow M, Quarg P, Spiegel R: Disease stage in Alzheimer disease and treatment effects of rivastigmine. Alzheimer Dis Assoc Disord 2004;18:123-128.

-12 Erkinjuntti T, Skoog I, Lane R, Andrews C: Potential long-term effects of rivastigmine on disease progression may be linked to drug effects on vascular changes in Alzheimer brains. Int J Clin Pract 2003;57:756-760.

-13 Kumar V, Anand R, Messina J, Hartman R, Veach J: An efficacy and safety analysis of Exelon in Alzheimer's disease patients with concurrent vascular risk factors. Eur J Neurol 2000;7:159-169.

14 Diagnostic Criteria from DSM-IV. Washington, American Psychiatric Association, 1994.

-15 McKhann G, Drachman D, Folstein M, Katzman R, Price D, Stadlan EM: Clinical diagnosis of Alzheimer disease: report of the NINCDS-ADRDA Work Group under the auspices of Department of Health and Human Services Task Force on Alzheimer's disease. Neurology 1984;34:939-944.

- 16 Folstein MF, Folstein SE, McHugh PR: 'Mini-mental state'. A practical method for grading the cognitive state of patients for the clinician. J Psychiatr Res 1975;12:189-198.

-17 Rosen WG, Mohs RC, Davis KL: A new rating scale for Alzheimer's disease. Am J Psychiatry 1984; 141:1356-1364.

- 18 Schneider LS, Olin JT, Doody RS, Clark CM, Morris JC, Reisberg B, Schmitt FA, Grundman M, Thomas RG, Ferris SH: Validity and reliability of the Alzheimer's Disease Cooperative Study-Clinical Global Impression of Change. The Alzheimer's Disease Cooperative Study. Alzheimer Dis Assoc Disord 1997;11(suppl 2):S22-S32.

-19 Galasko D, Bennett D, Sano M, Ernesto C, Thomas R, Grundman M, Ferris S: An inventory to assess activities of daily living for clinical trials in Alzheimer's disease. The Alzheimer's Disease Cooperative Study. Alzheimer Dis Assoc Disord 1997;11(suppl 2):S33-S39. 
-20 Lefèvre G, Sędek G, Jhee S, Leibowitz M, Huang H-LA, Enz A, Maton S, Pommier F, Schmidli H, Appel-Dingemanse S: Pharmacokinetics and pharmacodynamics of the novel daily rivastigmine transdermal patch compared with twice-daily capsules in Alzheimer's disease patients. Clin Pharmacol Ther 2008;83:106-114.

- 21 Erkinjuntti T, Skoog I, Lane R, Andrews C: Rivastigmine in patients with Alzheimer's disease and concurrent hypertension. Int J Clin Pract 2002;56:791-796.

-22 Venneri A, Shanks MF, Staff RT, Pestell SJ, Forbes KE, Gemmell HG, Murray AD: Cerebral blood flow and cognitive responses to rivastigmine treatment in Alzheimer's disease. Neuroreport 2002;13: 83-87.

-23 Venneri A: Imaging treatment effects in Alzheimer’s disease. Magn Reson Imaging 2007;25:953-968. 